\title{
On a Sufficient and Necessary Condition for Graph Coloring
}

\author{
Maodong Ye \\ Department of Mathematics, Zhejiang University, Hangzhou, China \\ Email: ymd@css.zju.edu.cn
}

Received August 5, 2013; revised September 3, 2013; accepted October 2, 2013

Copyright (C) 2014 Maodong Ye. This is an open access article distributed under the Creative Commons Attribution License, which permits unrestricted use, distribution, and reproduction in any medium, provided the original work is properly cited. In accordance of the Creative Commons Attribution License all Copyrights (C) 2014 are reserved for SCIRP and the owner of the intellectual property Maodong Ye. All Copyright (C) 2014 are guarded by law and by SCIRP as a guardian.

\section{ABSTRACT}

Using the linear space over the binary field that related to a graph $G$, a sufficient and necessary condition for the chromatic number of $G$ is obtained.

\section{KEYWORDS}

\section{Vertex Coloring; Chromatic Number; Outer-Kernel Subspace; Plane Graph}

\section{Introduction}

Let $G=(V, E)$ be a graph, where $V$ is a set of vertices and $E$ is a set of edges of $G$. A vertex coloring of a graph $G$ is a coloring to all the vertices of $G$ with $p$ colors so that no two adjacent vertices have the same color. Such the graph is called $p$-coloring. The minimal number $p$ is called the chromatic number of $G$, and is denoted by $\chi(G)$. The so-called Four Color Problem is that for any plane graph $G, \chi(G) \leq 4$ [1].

The coloring of a graph $G$ is an interesting problem for many people [2]. This is mainly caused by the Four Color Problem [3].

In this paper, putting a graph into a linear space over the binary field $G F(2)$, we obtain the sufficient and necessary condition for the chromatic number of $G$.

And as an application of above result, we give a characterization for a maximal plane graph to be 4-coloring.

\section{The Linear Space $A_{n}$ over GF(2)}

Now we introduce the linear space over the field $G F(2)$.

Firstly, the field $G F(2)$ contains only two members: $G F(2)=\{0,1\}$, where the addition and multiplication are as usual excepting that $1+1=0$.

Let $V_{n}=\left\{a_{1}, a_{2}, \cdots, a_{n}\right\}$ be the $n$ vertices, the all vectors of the linear space $A_{n}$ are formed of the symbolic expression

$$
\sum_{i=1}^{n} \alpha_{i} a_{i}, \quad \alpha_{i} \in G F(2)
$$

It has $2^{n}$ vectors. The addition of two vectors is defined by

$$
\sum_{i=1}^{n} \alpha_{i} a_{i}+\sum_{i=1}^{n} \beta_{i} a_{i}=\sum_{i=1}^{n}\left(\alpha_{i}+\beta_{i}\right) a_{i}
$$

Here, the $n$ vertices $\left\{a_{1}, a_{2}, \cdots, a_{n}\right\}$ will serve as the most basic elements of the linear space $A_{n}$. They will be as a basis of the linear space $A_{n}$. For them the basic assumption is that these $n$ vertices are linearly independent in $A_{n}$. 
According to the addition in $G F(2)$, for any vector $u$ in the linear space $A_{n}$, it has

$$
u+u=0 .
$$

Here we denote the zero vector by 0 .

For a vector $u=\sum_{i=1}^{n} \alpha_{i} a_{i}$, the order of the vector $u$ is defined by

$$
|u|=\sum_{i=1}^{n}{ }^{\prime} a_{i},
$$

where the $\sum^{\prime}$ means that the addition is the usual addition in the integer set.

A vector with $\mathrm{k}$ order is called a k-order vector, and a vector whose order is even is called an even-order vector.

We now give some structures to the linear space $A_{n}$. In other words, we want to "put" a graph into the linear space $A_{n}$.

In the linear space $A_{n}$, 1-order vectors are vertices of a graph. The edge is expressed as the 2-order vertex, i.e. $a_{i}+a_{j}$ is the edge $a_{i} a_{j}$. So we have two ways to describe a edge: by $a_{i} a_{j}$ (in the usual sense), or by $a_{i}+a_{j}$ (in the linear space $A_{n}$ ).

In the following, we always discuss a graph in the linear space $A_{n}$, it means we express edges with the second form.

All the 2-order vectors in the linear space $A_{n}$ are the all possible edges with $n$ vertices $\left\{a_{1}, a_{2}, \cdots, a_{n}\right\}$, that we denote by $E_{\mathrm{A}}$ :

$$
E_{A}=\left\{a_{i}+a_{j}, i \neq j, i, j \in\{1, \cdots, n\}\right\} .
$$

For a giving graph $G=\left(V_{n}, E\right)$ with $n$ vertices, in the linear space $A_{n}$, the elements of the set $E$ are the 2-order vectors of $A_{n}$, then the edge set $E$ of $G$ is the subset of the set $E_{A}, E \subseteq E_{A}$.

We give two examples here.

1) For the set $E_{A}$ with all the 2-order vertices in $A_{n}$, the graph $K_{n}=\left(V_{n}, E_{A}\right)$ is a complete graph, whose any two vertices are adjacent.

2) For a graph $G=\left(V_{n}, E\right)$ with $n$ vertices, the complementary set of $E$ in the set of the 2-order vertices of $A_{n}$ is $E_{A} \backslash E$. Then the complementary graph $\hat{G}$ of the graph $G$ is $\hat{G}=\left(V_{n}, E_{A} \backslash E\right)$.

We now see the addition in $A_{n}$. For a path of $G$ with a sequence of edges $a+b_{1}, b_{1}+b_{2}, \cdots, b_{k}+b$, where the end-points are $a$ and $b$, the sum of the edges is:

$$
\left(a+b_{1}\right)+\left(b_{1}+b_{2}\right)+\cdots+\left(b_{k}+b\right)=a+b .
$$

This expression indicates the relation between the addition in the linear space and the connectivity of a graph. That is why we put the graph into the linear space $A_{n}$.

Lemma 1. The sum of even-order vectors is even-order.

This is clear by the property that $a_{i}+a_{j}=0$ if and only if $i=j$.

As a special case of the Lemma 1 , we have

Lemma 2. Let $a_{i_{j}} \neq 0,(j=1,2, \cdots, k)$ are the vertices of $G$, if

$$
a_{i_{1}}+a_{i_{2}}+\cdots+a_{i_{k}}=0,
$$

then $k$ is even.

Definition. Let $A_{n}$ be the $n$-dimensional linear space derived by the graph $G=\left(V_{n}, E\right)$ above, and $R_{G} \subseteq E_{A}$ be a set of 2-order vectors. Denote by $\bar{R}_{G}$ the linear subspace spanned by $R_{G}$. If there are no edges of $E$ in $\bar{R}_{G}$, i.e.

$$
\bar{R}_{G} \cap E=\phi
$$

then $\bar{R}_{G}$ is called an outer-kernel subspace of $G$. And $\bar{R}_{G}$ is a maximal outer-kernel subspace if the rank of $\bar{R}_{G}$ is maxima in all the outer-kernel subspace of $G$.

Now we give some basic properties of a outer-kernel subspace of a graph $G$.

By definition, $\bar{R}_{G}$ is a subspace of $A_{n}$. Denote the set of all 2-order vectors of $\bar{R}_{G}$ by $E\left(\bar{R}_{G}\right)$, then $G\left(\bar{R}_{G}\right)=\left(V_{1}, E\left(\bar{R}_{G}\right)\right)$ is a subgraph of the complementary graph $\hat{G}$ of $G$, here $V_{1}$ is the 1-order vectors 
appeared in $E\left(\bar{R}_{G}\right)$. The subgraph $G\left(\bar{R}_{G}\right)$ consists of some connected blocks.

Lemma 3. Let $g_{1}=\left(H, E_{1}\right)$ be a connected block of $G\left(\bar{R}_{G}\right)$, and $H=\left\{a_{1}, a_{2}, \cdots, a_{m}\right\}$ be the vertices of $g_{1}$, then $m \geq 2, g_{1}$ is a complete graph $K_{m}$ in the $G\left(\bar{R}_{G}\right)$ and

is the linear subspace of $\bar{R}_{G}$

$$
B=\operatorname{span}\left\{a_{1}+a_{2}, a_{2}+a_{3}, \cdots, a_{m-1}+a_{m}\right\}
$$

This lemma means that every connected block of $G\left(\bar{R}_{G}\right)$ is a complete graph.

Proof. Because $\bar{R}_{G}$ is spanned by 2-order vectors, so $m \geq 2$.

Suppose that $a_{i}+a_{j} \in E_{1}, a_{j}+a_{k} \in E_{1}$, for $\bar{R}_{G}$ is a linear space, then

$$
\left(a_{i}+a_{j}\right)+\left(a_{j}+a_{k}\right)=a_{i}+a_{k} \in \bar{R}_{G} .
$$

Since $g_{1}$ is connected block of $G\left(\bar{R}_{G}\right)$, so $a_{i}+a_{k} \in E_{1}$. On the other hand, if $a_{i}+a_{j} \in E_{1}$, then $a_{i}, a_{j} \in H$. Hence all the 2-order vectors formed by the set of vertices $H$ span the linear subspace $B$ of $\bar{R}_{G}$. Thus the connected block $g_{1}$ is a complete graph in $g\left(\bar{R}_{G}\right)$.

Lemma 4. If $a_{1}+a_{2}+\cdots+a_{k} \in \bar{R}_{G}$, then $k$ is even and there exists a $i \in\{2, \cdots, k\}$ such that $a_{1}+a_{i} \in \bar{R}_{G}$.

Proof. By the definition of $\bar{R}_{G}, k$ is even. For $\bar{R}_{G}$ is spanned by 2-order vectors, so $a_{1}$ is in a connected block $K_{m}$ of $\bar{R}_{G}$. Thus another vertex $a_{i}(i \neq 1)$ of $K_{m}$ must appear in $a_{1}+a_{2}+\cdots+a_{k}$.

\section{The Main Results}

The outer-kernel subspace plays an important role in the problem of vertex coloring.

Theorem 1. Let $G_{n}$ be a graph with $n$ vertices, then the sufficient and necessary condition for $G_{n}$ to be $p$-coloring is that the rank of an outer-kernel subspace $\bar{R}_{G}$ of $G_{n}$ is $n-p$.

Proof. First we prove the necessity. Suppose that the graph $G_{n}$ is $p$-coloring. Then all the vertices of $G_{n}$ can be divided into $p$ subsets by the colors:

$$
S_{1}, S_{2}, \cdots, S_{k}, Q_{k+1}, \cdots, Q_{p}, k \leq p .
$$

That means the vertices with a same color are in the same subset. Because it may have a subset with only one vertex, we denote the one-vertex subsets with different colors by $Q_{k+1}, \cdots, Q_{p}$.

The elements of subset $S_{i}(i=1,2, \cdots, k)$ are not less then 2 . Denote them by

$$
S_{i}=\left\{a_{i 1}, a_{i 2}, \cdots, a_{i t_{i}}\right\}, t_{i} \geq 2,
$$

then by (2)

$$
\sum_{i=1}^{k} t_{i}+p-k=n
$$

Let

$$
E_{i}=\left\{a_{i 1}+a_{i 2}, a_{i 1}+a_{i 3}, \cdots, a_{i 1}+a_{i t_{i}}\right\}, \quad i=1,2, \cdots, k,
$$

and

$$
R_{G}=E_{1} \cup E_{2} \cup \cdots \cup E_{k},
$$

then the vectors of $R_{G}$ are independent. Hence by (3), the dimension of subspace $\bar{R}_{G}$ spanned by $R_{G}$ is

$$
\operatorname{dim} \bar{R}_{G}=\sum_{i=1}^{k} t_{i}-k=n-p
$$

It is clear that $\bar{R}_{G} \cap E=\phi$.

For the sufficiency, suppose that there exists an outer- kernel subspace $\bar{R}_{G}$ with condition (1), and the dimension of $\bar{R}_{G}$ is $n-p$.

We divide the vertices of $G$ into some subsets according to the subspace $\bar{R}_{G}$. If for two vertices $a$ and $b$ there have

$$
a+b \in \bar{R}_{G},
$$

then we put $a$ and $b$ into a same subset. Like the notation of congruence we denote

$$
a \equiv b\left(\bmod \bar{R}_{G}\right) \text {. }
$$


Obviously, if a vertex $a$ appears in $\bar{R}_{G}$, then there has at least another vertex in the same subset with $a$. If a vertex does not appear in $\bar{R}_{G}$, then this vertex forms a subset by itself, i.e. the subset contains only one vertex.

Lemma 5. The vertices from different subset are linear independence on $\bar{R}_{G}$, i.e. if $a_{1}, a_{2}, \cdots, a_{m}$ belong to different subsets respectively, then

$$
a_{1}+a_{2}+\cdots+a_{m} \equiv 0\left(\bmod \bar{R}_{G}\right) .
$$

In fact, if $a_{1}+a_{2}+\cdots+a_{m} \in \bar{R}_{G}$, by Lemma 4, there exists a vertex $a_{i} \in\left\{a_{2}, \cdots, a_{m}\right\}$ such that $a_{1}+a_{i} \in \bar{R}_{G}$. That means $a_{1}, a_{i}$ is in the same subset.

Now we go on with the proof of the sufficiency. Suppose that the 2-order vectors $r_{1}, r_{2}, \cdots, r_{n-p}$ form a basis of $\bar{R}_{G}$, and the vertices of the graph $G$ are now divided into the disjoint subset $N_{1}, N_{2}, \cdots, N_{l}$ by the method above. Take $b_{i} \in N_{i}, \quad i=1,2, \cdots, l$, then any vertex $a$ of $G$ must be in some subset $N_{i}$ and by (5) we have

$$
a=b_{i}+r, r \in \bar{R}_{G} \text {. }
$$

So any vertex of $G$ can be expressed by $b_{i}$ and an element of $\bar{R}_{G}$. Thus by Lemma 5,

$$
b_{1}, b_{2}, \cdots, b_{1}, r_{1}, r_{2}, \cdots, r_{n-p}
$$

are the basis of linear space $A_{n}$. Hence $l=p$.

By the definition of $\bar{R}_{G}$ and (5) we know that the two vertices in the same subset $N_{i}$ are non-adjacent. Thus, we can assign one color to the vertices of each subset $N_{i}$. So we just need $p$ colors for $G$. The graph is $p$-coloring.

Due to Theorem 1 and the expression (4), we have:

Theorem 2. For a graph $G$ with $n$ vertices, the sufficient and necessary condition for $\chi(G)=p$ is that the rank of a maximal outer-kernel subspace $\bar{R}_{G}$ is $n-p$.

\section{An Application to Plane Graphs}

As an application of Theorem 1, we consider a result of the coloring to the plane graph.

A maximal plane graph is a graph $G$ such that for any two non-adjacent vertices $a$ and $b$ of $G, G$ added to the edge $a b$ makes a non-planar graph. It is clear that all the faces of a maximal plane graph are triangles.

A maximal plane graph is 3-CR-edge coloring if we can color its edges by 3 colors such that the three edges of every its triangle face are coloring by different colors. Later we will see that the CR in the definition is borrowed from the Cauchy-Riemann condition in the complex function theory.

Theorem 3. If a maximal plane graph is 3-CR-edge coloring, then the graph is 4-vertex coloring.

The inverse of the Theorem 3 is true, too. That means if a maximal plane graph is 4-vertex coloring, then the graph is 3-CR-edge coloring.

Proof. We introduce the 2-demensional linear space $A_{2}$ :

$$
A_{2}=\{(0,0),(0,1),(1,0),(1,1)\} .
$$

Let $G=\left(V_{n}, E\right)$ be 3-CR-edge coloring, and the all edges of $G$ can map to the three elements $(0,1),(1,0),(1,1)$ of $A_{2}$ by their colors, respectively. That is the mapping $f$

$$
f: E \rightarrow A_{2}
$$

such that if $a, b, c$ are the vertices of a face of $G$, then

$$
f(a+b)+f(b+c)+f(c+a)=0
$$

For a path of $G$ with the end-point $a, b$ and the sequence of the edges $a+a_{i_{1}}, a_{i_{1}}+a_{i_{2}}, \cdots, a_{i_{k}}+b$, we define

$$
f(a+b)=f\left(a+a_{i_{1}}\right)+f\left(a_{i_{1}}+a_{i_{2}}\right)+\cdots+f\left(a_{i_{k}}+b\right) .
$$

By the condition of 3-CR-edge coloring, the extending mapping $f$ by (7) is dependent only on the end-point $a$ and $b$, and independent on their path.

Let $A_{n}^{\prime}$ be the $(n-1)$-dimensional linear subspace spanned by all the 2-order vectors of the space $A_{n}$. Then $f$ is the homomorphic mapping from subspace $A_{n}^{\prime}$ onto the space $A_{2}$. The homomorphic kernel $\bar{R}$ consists of such vector $e$ of $A_{n}^{\prime}$ that satisfies 


$$
f(e)=0
$$

Suppose $R$ is the subset of 2-order vectors of $A_{n}^{\prime}$ that satisfies (8) and $\bar{R}$ is spanned by $R$. Then by (8) we have

$$
\bar{R} \cap E=\phi .
$$

Denote the linear independent spanning elements of $R$ by $e_{1}, e_{2}, \cdots, e_{m}$, that is just the basis of $\bar{R}$. And $\operatorname{dim} \bar{R}=m$.

We take $e_{\alpha}, e_{\beta}, e_{\gamma} \in E$ such that

$$
f\left(e_{\alpha}\right)=(0,1), f\left(e_{\beta}\right)=(1,0), f\left(e_{\gamma}\right)=(1,1) .
$$

Then the linear subspace $A_{n}^{\prime}$ is spanning by

$$
e_{\alpha}, e_{\beta}, e_{\gamma}, e_{1}, e_{2}, \cdots, e_{m} .
$$

Hence $m+3 \geq n-1$, and $\operatorname{dim} \bar{R}=m \geq n-4$. By Theorem 1, the graph $G$ is 4 -vertex coloring.

\section{REFERENCES}

[1] F. Harary, “Graph Theory,” Addison-Wesley, Boston, 1969.

[2] P. Erdös and A. Hajnal, “Chromatic Number of Finite and Infinite Graphs and Hypergraphs,” Discrete Mathematics, Vol. 53, 1985, pp. 281-285.

[3] N. Biggs, E. Lloyd and R. Wilson, “Graph Theory 1736- 1936,” Oxford University Press, Oxford, 1986. 